\title{
Waves and instabilities in rotating free surface flows
}

\author{
J. MougeL ${ }^{1, a}$, D. FABre ${ }^{1}$ And L. LACAZE ${ }^{1,2}$ \\ 1 Institut de Mécanique des Fluides de Toulouse, UMR CNRS/INPT/UPS 5502, Allée Camille Soula, 31400 Toulouse, France \\ 2 CNRS, IMFT, 31400 Toulouse, France
}

Received 5 July 2013, Accepted 20 January 2014

\begin{abstract}
The stability properties of the rotating free surface flow in a cylindrical container is studied using a global stability approach, considering successively three models. For the case of solid body rotation (Newton's bucket), all eigenmodes are found to be stable, and are classified into three families: gravity waves, singular inertial modes, and Rossby waves. For the case of a potential flow, an instability is found. The mechanism is explained as a resonance between gravity waves and centrifugal waves, and is thought to be at the origin of the "rotating polygon instability" observed in experiments where the flow is driven by rotation of the bottom plate (see L. Tophøj, J. Mougel, T. Bohr, D. Fabre, The Rotating Polygon Instability of a Swirling Free Surface Flow, Phys. Rev. Lett. 110 (2013) 194502). Finally, in the case of the Rankine vortex which in fact consists in the combination of the two first cases, we report a new instability mechanism involving Rossby and gravity waves.
\end{abstract}

Key words: rotating flows / waves / instabilities

\section{Introduction}

Rotating flows with a free surface are of major importance in a large range of fields ranging from geophysics to many industrial applications. These flows are known to support rich dynamics and spectacular examples are provided by a very simple experiment consisting of a cylindrical container partly filled with a liquid, in which the fluid motion is driven by a rapidly rotating bottom while the side wall is maintained fixed (see Fig. 1a). For high enough rotation rates, a symmetry breaking is observed, leading to the formation of polygonal patterns rotating at a constant angular velocity on the free surface. This phenomenon, first observed in [2], has been revisited in [3-6] and the experimental patterns observed by [5] are reproduced here on Figure 1b.

The objective of this paper is to give a brief summary of a research effort, conducted along the past two years, aiming at explaining those polygonal patterns as resulting from a linear instability of the axisymmetric flow predating them. For this purpose, we developed a global stability approach which allows to study the linear dynamics of various models of free surface swirling flows. After presenting the general method, we will review the results obtained with three successive models. First, in the case of a solid-body rotation, we observe only stable modes. However, the linear dynamics of this flows turn

\footnotetext{
${ }^{\mathrm{a}}$ Corresponding author: jerome.mougel@imft.fr
}

out to be rather interesting, as it involves singular modes displaying analogy with some kinds of inertial waves existing in rotating stars and spherical shells [7], and Rossby waves similar to those existing in a closed container with a non-flat bottom. Secondly, in the case of a potential rotation, we report the existence of an instability mechanism existing for all azimuthal wavenumbers $m \geq 2$. The mechanism is explained as a resonance between gravity waves and centrifugal waves, and was recently proposed to be at the origin of the aforementionned polygonal patterns [1]. Finally, we will show preliminary results obtained using an improved model combining an inner region in solidbody rotation and an outer region in potential rotation (Rankine model).

\section{General equations}

Using cylindrical coordinates $(r, \theta, z)$, we consider a general azimuthal base flow of the form $\mathbf{U}_{\mathbf{0}}=U_{\theta}(r) \mathbf{e}_{\theta}$. The flow occupies a domain defined by $0<r<R$ and $0<z<h(r)$, where $R$ is the radius of the cylindrical tank, and $h(r)$ is the altitude of the free surface. Neglecting the effect of surface tension, the shape of the free surface is related to the flow through the equation $h^{\prime}=g_{c} / g$ where $h^{\prime}$ is the derivative of $h$ with respect to $r, g$ is the gravity and $g_{c}=U_{\theta}^{2} / r$ is the centrifugal acceleration (this condition means that the free surface is perpendicular to the total acceleration $\left.-g \mathbf{e}_{\mathbf{z}}+g_{c} \mathbf{e}_{\mathbf{r}}\right)$. 


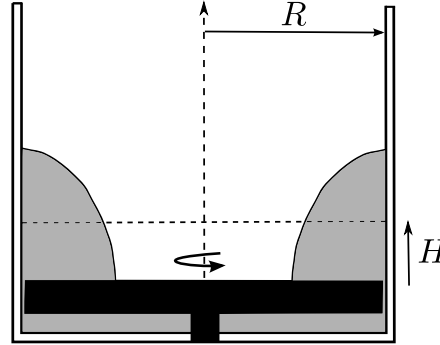

(a)

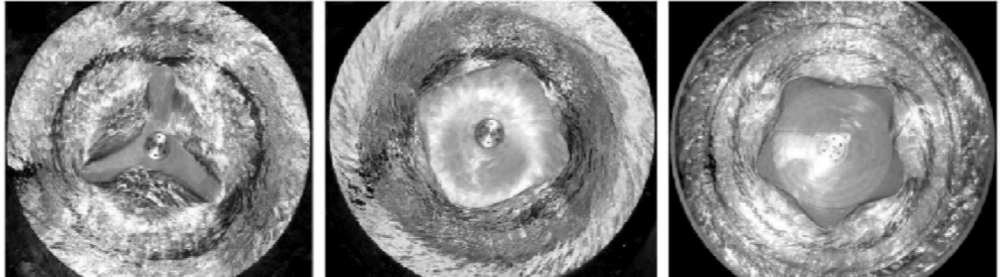

(b)

Fig. 1. (a) Experimental configuration: rotating bottom plate (black); fixed outer wall (white); fluid, typically water (grey). (b) Experimental visualisations from [5] displaying typical polygonal shapes.

To investigate the stability of such flows we add small amplitude perturbations, under modal form, i.e. $\epsilon[u, v, w, p, \eta] \exp i(m \theta-\omega t)$, with $m$ the azimuthal wave number and $\omega$ the complex frequency. The eigenmode structure has velocity and pressure $[u, v, w, p]$ components defined in the bulk (as function of $r$ and $z$ ) while the component $\eta$ corresponding to the vertical free surface displacement is defined on the boundary of the domain (function of $r$ only). For sake of simplicity we absorb the constant density in the pressure component. Restricting to the inviscid case $^{1}$, the linearized equations governing the dynamics of the perturbations are obtained from the Euler and continuity equations :

$$
\begin{aligned}
-i\left(\omega-m \frac{U_{\theta}}{r}\right) u & =-\frac{\partial p}{\partial r}+2 \frac{U_{\theta}}{r} v, \\
-i\left(\omega-m \frac{U_{\theta}}{r}\right) v & =-\frac{i m}{r} p-\frac{1}{r} \frac{d\left(r U_{\theta}\right)}{d r} u, \\
-i\left(\omega-m \frac{U_{\theta}}{r}\right) w & =-\frac{\partial p}{\partial z}, \\
\frac{\partial u}{\partial r}+\frac{u}{r}+\frac{i m}{r} v+\frac{\partial w}{\partial z} & =0 .
\end{aligned}
$$

On the bottom and side walls we apply non-penetration boundary conditions. At the free surface, we obtain linearized dynamic and kinematic boundary conditions of the form :

$$
p-g \eta=0 ; \quad-i\left(\omega-m \frac{U_{\theta}}{r}\right) \eta=w-h^{\prime} u .
$$

In the following we impose an analytical form for $U_{\theta}$ and perform a global stability analysis of this particular flow. In the finite element framework equations (1) and (2) can be written under the form of a generalized eigenvalue problem $A X=\omega B X$ with $\omega$ the eigenvalue, $X$ the corresponding eigenmode and $A$ and $B$ complex matrices constructed with the finite element software FreeFem $++[9]$. We note $\omega=\omega_{r}+i \omega_{i}$, where $\omega_{r}$ will refer to the oscillation frequency and $\omega_{i}$ to the growth rate. In particular $\omega_{i}>0$ will denote instability.

1 The linearized equations in the general case, retaining viscosity and surface tension, are given in [8].

\section{Solid body rotation (Newton's Bucket)}

The first case under investigation corresponds to a solid rotation of the whole liquid, corresponding to an azimuthal velocity profile of the form $U_{\theta}=r \Omega$. This flow can be parametrized by a Froude number $F=\Omega \sqrt{R / g}$ and a gravitational Reynolds number $C^{-1}=R^{3 / 2} \sqrt{g} / \nu$. It is a classical exercise to show that the free surface is parabolic. Assuming that the volume of liquid is conserved and equal to $\pi R^{2} H$, where $H$ is the liquid height at rest, one can further show that the bottom remains "wet" for $F<2 \sqrt{H / R}$ and gets "dry" above this threshold (see Fig. 2a).

In the inviscid case one can reduce equations for the perturbation in the bulk (1) to a single equation for pressure which is known as Poincaré's equation:

$$
\frac{\partial^{2} p}{\partial r^{2}}+\frac{1}{r} \frac{\partial p}{\partial r}-\frac{m^{2}}{r^{2}} p+\left(\frac{(\omega-m \Omega)^{2}-4 \Omega^{2}}{(\omega-m \Omega)^{2}}\right) \frac{\partial^{2} p}{\partial z^{2}}=0 .
$$

A well-known feature of this equation is that the nature of the possible solutions change drastically depending on the relative frequency (with respect to the rotating frame) $\omega-m \Omega$ (see [10] for instance). Three cases have to be considered.

First, if $|\omega-m \Omega|>2 \Omega$, the equation is elliptic, and regular eigenmode solutions are expected to exist. Numerical results from the global stability analysis show that this is effectively the case, and Figure $2 b$ shows that the solutions organize into two sets of branches. Figure $3 \mathrm{a}$ displays the typical structure of the corresponding eigenmodes : the structure is regular and the maximum pressure levels are located along the free surface. These modes are thus recognized as gravity waves, and in the limit of vanishing rotation $(F \approx 0)$ their frequencies match with those of the pure sloshing modes in a cylindrical container with flat surface [11].

Secondly, if $|\omega-m \Omega|$ is smaller than $2 \Omega$ (but not too small compared to $\Omega$ ), the Poincaré equation becomes hyperbolic. In such cases, regular eigenmode solutions are not expected to be met (except in the limit of small rotation where the surface becomes flat, and a separation of variables is possible). Instead, computations in the strictly inviscid case reveal the existence of a dense set of eigenfunctions corresponding to singular inertial modes. The 


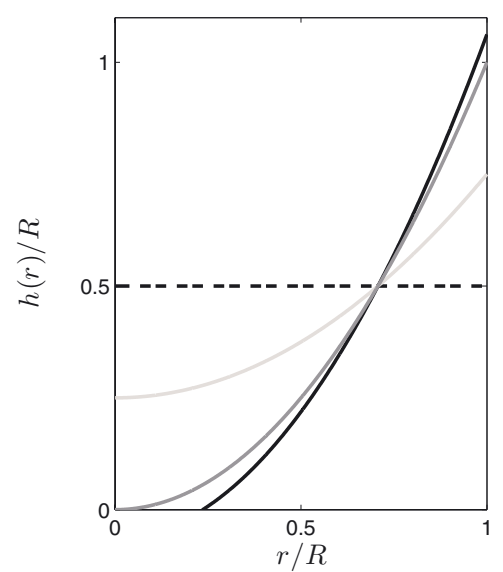

(a)

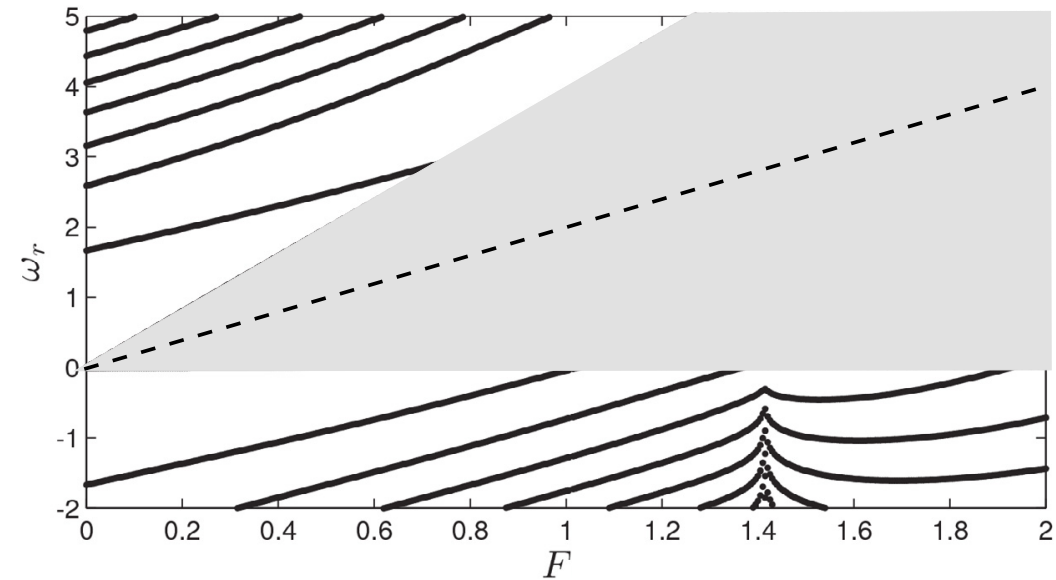

$(b)$

Fig. 2. Solid body rotation (with $H / R=0.5$ ) : (a) shape of the free surface for $F=[1, \sqrt{2}, 1.5]$. (b) Oscillation rates of the normal modes as function of $F, m=2$ (inviscid case). The black lines correspond to the regular gravity waves; the grey area indicates the range of existence of the singular inertial modes, and the dashed line corresponds to $\omega=m \Omega$, in the vicinity of which the Rossby waves are found.

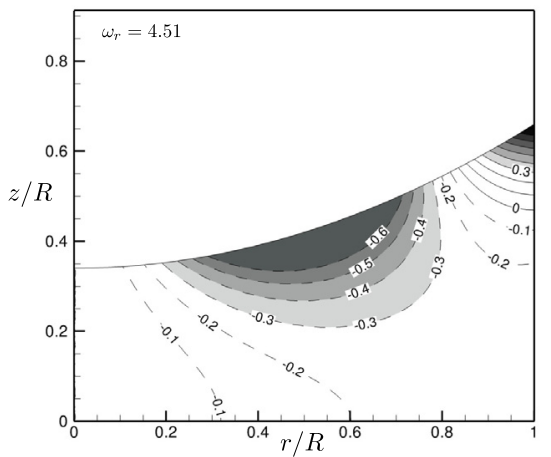

(a)

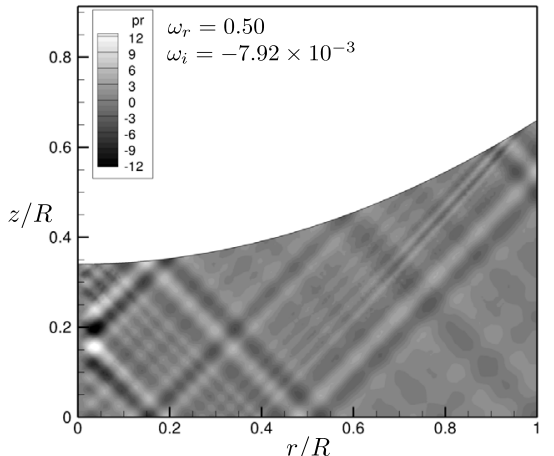

(b)

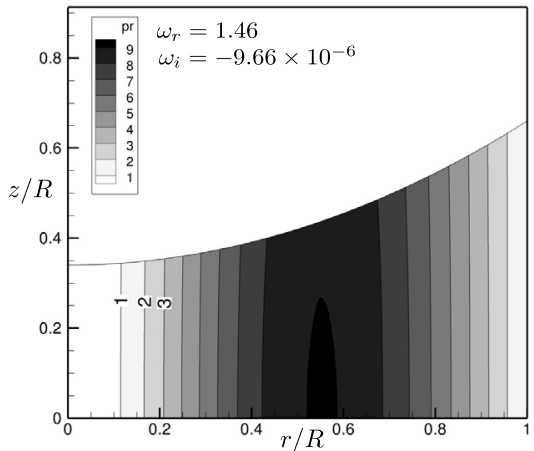

(c)

Fig. 3. Solid-body rotation $(H / R=0.5)$ : structure in the meridional plane of some eigenmodes (pressure component $\operatorname{Re}[p(r / R, z / R) / p(1, h(1))])$ obtained for $F=0.8, H / R=0.5, m=2$. (a) Regular gravity wave (inviscid case). (b) Singular inertial mode with ray pattern. (c) Rossby wave. (plots (b) and (c) are viscous results with $C^{-1}=10^{-7}$; here viscosity is introduced into the bulk equations only for sake of regularization).

range of existence of these singular eigenmodes is indicated by the shaded area in Figure 2b. The singularity can be regularized by introducing a small amount of viscosity. Figure $3 \mathrm{~b}$ displays the structure of a viscous mode obtained in this way (see [8] for details about the numerical implementation of the viscous case). Such modes are found to exhibit a striking ray-like structure, similar to the patterns observed in other configurations such as spherical shells [7], or rotating stars.

The third interesting case is met if the relative frequency $|\omega-m \Omega|$ is close to zero, i.e. when the perturbations are almost stationnary with respect to the rotating frame. In this case, the Poincaré equation (3) becomes degenerate, and the Euler equations (1) reduce, at leading order, to the geostrophic equilibrium. In such a quasigeostropic context, variations of the height of liquid is known to allow for the possibility of a type of slow waves called Rossby waves. Such solutions are well-known in the case where the variation of height is due to a non-flat bottom (see [10], p. 85 for instance). In the present case, variation of height is also present because of the parabolic shape of the free surface, and the numerical results effectively demonstrates the existence of such Rossby waves. An example of such a wave is displayed in Figure 3c. As can be seen, the structure of the mode (as displayed by the pressure component) is nearly independent of the vertical direction, which is a characteristic of flow motion in the quasi-geostrophic limit.

Note that as the rotation is increased, Figure 2b indicates that some of the branches of gravity waves enter the "hyperbolic" interval. Complex interactions between the three kinds of modes are expected in this case. Another interesting feature is the discontinuity met along the branches of gravity waves at the wet/dry transition for $F=2 \sqrt{H / R}$. These features are the object of current investigations. 
(a)

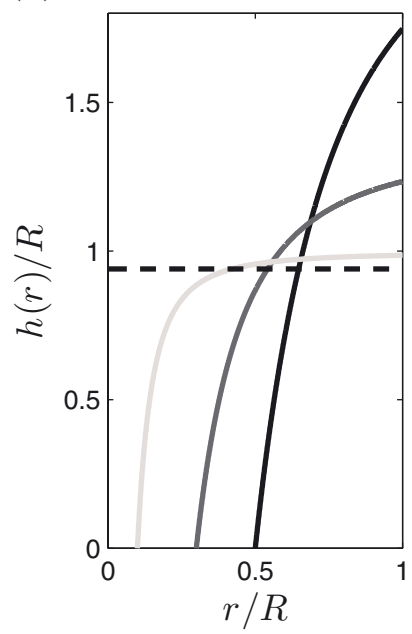

(b)
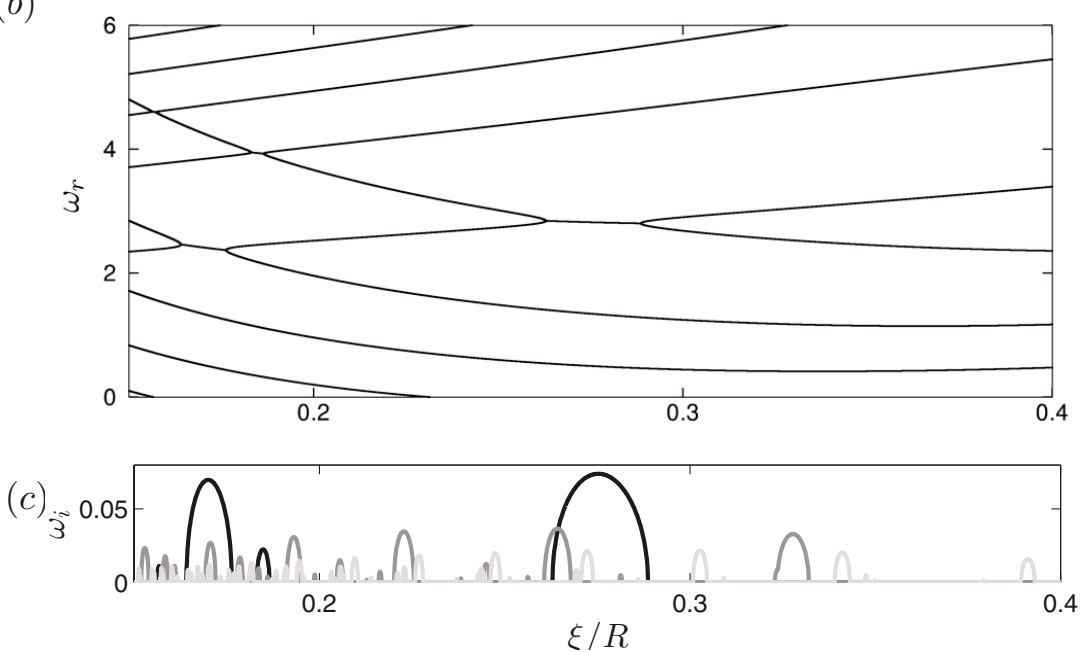

Fig. 4. Potential rotation (with $H / R=0.94$ ): (a) shape of the free surface for $\xi / R=[0.1,0.3,0.5]$. (b) Oscillation rates $\omega_{r}$ for $m=2$. (c) Growth rate $\omega_{i}$ for $m=2$ (black), $m=3$ (dark grey) and $m=4$ (light grey).

\section{Potential rotation}

Although the solid-body rotation model considered above seems the most natural model for the flow in a cylinder with a rotating bottom, theoretical arguments as well as experimental observations using PIV (see [3]) actually indicate that the potential rotation is a more appropriate model, especially in the cases with high rotation rate. We thus consider, as our second model, a potential model defined as $U_{\theta}(r)=\Gamma /(2 \pi r)$ where $\Gamma$ is the circulation. In this case the free surface is concave, with expression $h(r)=\Gamma^{2} /\left(8 \pi^{2} g\right)\left(1 / \xi^{2}-1 / r^{2}\right)$, valid for $r>\xi$, where $\xi$ is the radius of the central 'dry' region (see Fig. 4a). The parameters $\Gamma$ and $\xi$ are related through the conservation of the volume of liquid, assuming again a total volume $\pi R^{2} H$ where $H$ is the liquid height at rest. Here we find more convenient to parametrize the results in terms of $\xi / R$ and $H / R$.

In the global stability analysis, if we restrict to the inviscid case, we can take advantage of the potential nature of the base flow and assume the perturbation to be potential as well, leading to great simplifications in the analysis. Such a global analysis was carried out in [1], were it was shown that the potential flow is generically unstable to perturbations with wavenumbers $m>1$.

In [1], we mostly focussed on the case $(H / R=0.276)$, which was documented in the experiments of [5]. In the present paper, we consider more particularly the case $(H / R=0.94)$, which corresponds to the experimental studies by [6]. In that experiment, only elliptical patterns $(m=2)$ have been observed. Figure 4c displays the amplification rates predicted by the global analysis in that case as function of $\xi / R$. It is found that instabilities with wavenumber $m=2$ are by far the most amplified ones in this case, suggesting that the potential instability mechanism could also be at the origin of the observed patterns. Note that in the experiment, the elliptical patterns were found to alternate temporally with axisymmetric states, a phenomenon called "surface switching". This phenomenon is certainly non linear and thus cannot be predicted by our approach.

Figure $4 \mathrm{~b}$ displays the real part of the frequency (for the same conditions as in Fig. 4c), restricting to the case $m=2$. This figure illustrates the fact that instabilities arise in ranges of $\xi$ corresponding to the crossing between two families of branches. In reference [1], we argued that these two families of branches actually correspond to two different kinds of surface waves: the branches going upwards are gravity waves, since the restoring force responsible for them is the gravity acting on the external part of the free surface where it is nearly horizontal, while the branches going downwards are centrifugal waves since in their case the restoring force is the centrifugal effect acting on the inner part of the free surface where it is nearly vertical.

For particular values of $\xi$ we see that two waves of each type can resonate. It has been shown that this resonant behavior leads either to an instability creating two complex conjugate modes during the merging, or to a stable no-merging process where both waves exchange identities. The selection of the crossing process is related to the energy of both waves as defined in [12] and an instability can be found only if one wave has negative energy and the other positive energy (here a wave having negative energy means that the wave+base flow system has less energy than the base flow). In reference [1] it is found using Cairns terminology (see [12]) and a simple $2 \mathrm{D}$ model that the energy of the gravity and centrifugal waves respectively read $E_{g} \sim\left(\omega-m U_{\theta}(R) / R\right)$ and $E_{c} \sim\left(\omega-m U_{\theta}(\xi) / \xi\right)$. These expressions mean that the centrifugal waves should be slower than the base flow and the gravity waves faster for the instability to occur (i.e. existence of a critical radius $r_{c} \in[\xi, R]$ where the global mode and the base flow have the same angular velocity). 

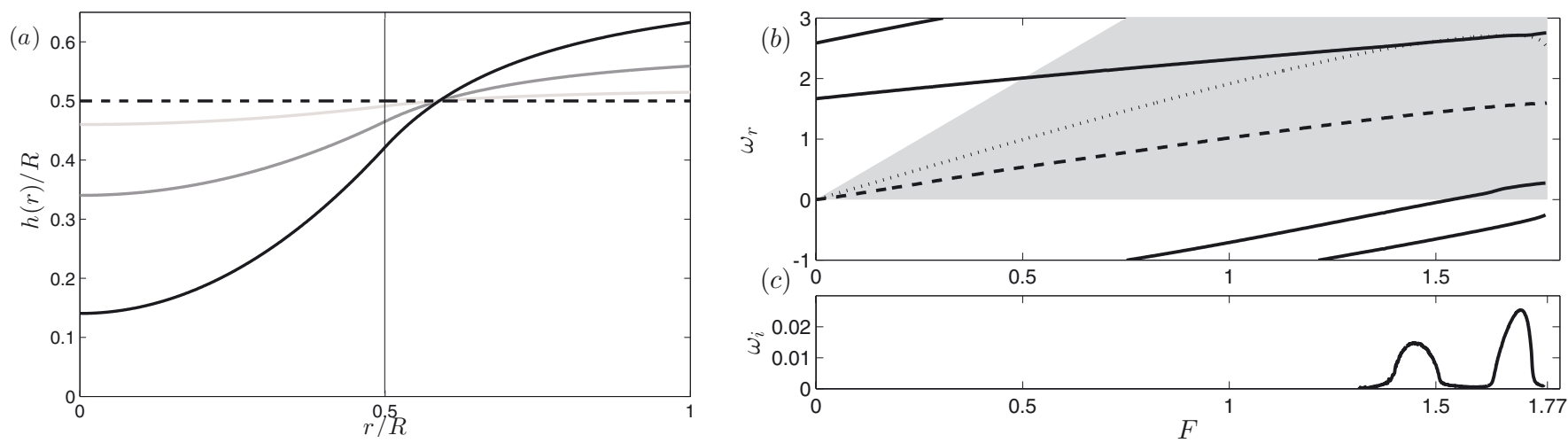

Fig. 5. Rankine model (with $H / R=a / R=0.5$ ): (a) shape of the free surface for $F=[0.5,1,1.5]$ (the vertical line separates the vortex core from the outer potential zone). (b) Oscillation frequencies $\omega_{r}$ of some of the wave solutions encountered in the global stability analysis for $C^{-1}=10^{-4}$ and $m=2$ (full lines: gravity waves; dotted line: Rossby wave; dashed line: regular Kelvin-Kirchhoff wave; grey area: range of existence of singular inertial waves). (c) Growth rate of the encountered unstable mode.

From Figure 4c, it can be noted that instability "bubbles" get much narrower when $m$ increases and in fact the maximum growth rate decreases rapidly. This fact is confirmed by an ongoing asymptotic study, conducted in the shallow water limit, which indicates a trend with the form $\omega_{i \max }=\sqrt{m} e^{-C m}$ with $C>0$. Thus the fact that no polygons with more than 6 corners are observed experimentally $[2,5]$ could simply mean that the theoretical instability obtained in the potential case is too weak to be observed in the real setup for these values of $m$.

\section{Rankine vortex}

A drawback of the potential model considered in the previous section is that the free surface necessarily touches the bottom at some location $r=\xi$ (see Fig. 4a), so it certainly cannot account for the "wet" states observed experimentally at low or moderate values of the rotation rate. Indeed, in such cases, the experimental results of [3] indicate that in the central region of the container, the motion is actually close to a solid-body rotation. This leads one to introduce an improved model combining an inner core in solid body rotation and an outer potential zone (Rankine vortex). The velocity of this base flow then reads

$$
U_{\theta}=\Omega r \text { for } 0<r \leqslant x, U_{\theta}=\frac{\Gamma}{2 \pi r} \text { for } x<r<R,
$$

with $a$ the radius of the vortex core, and $\Gamma=2 \pi \Omega a^{2}$ (assuming continuity at $r=x$ ). In the following we define the control parameter as $F=\Omega \sqrt{R / g}$, and we fix $H / R=0.5$ and $x / R=0.5$, a choice which is supported by recent results [13] suggesting that the radius of the inner core does not depend much upon the Froude number. Figure 5a displays the shape of the corresponding free surface. The boundary of the vortical core at $r=x$ is associated with a change of curvature of the free surface, from concave to convex. With the chosen parameters, the wet/dry transition occurs at $F \approx 1.77$.

Global stability results of this flow for $m=2$ are displayed in Figures 5b and 5c. Figure 5b depicts the oscillation frequencies of the modes as function of $F$. The results show some similarity with those obtained in the case of solid-body rotation (not surprisingly, since the Poincaré equation also holds in the inner zone in solid body rotation). As previously, we observe the existence of regular gravity waves (plain lines), a dense set of singular modes (grey area), and some Rossby-like waves in the range $\omega \approx m \Omega$ (dotted line). The model also leads to the existence of a new kind of wave with a different nature, whose frequency follows approximatively the trend $\omega \approx(m-1) \Omega$ (dashed line in Fig. 5b). Inspection of the structure of this wave shows that it corresponds to an oscillation of the boundary of the solid-body rotation core; allowing to identify it with the classical twodimensional oscillation mode of a vortex patch, also called the Kelvin-Kirchhoff (KK) wave (see [14]). In the sample case displayed in Figure 5c, we observe a double crossing between a gravity wave and a Rossby wave in the approximate range $F \approx[1.4,1.7]$. This interaction is unstable, and yields modes with positive amplification rates in this range (Fig. $5 \mathrm{c}$ ). Note that the stability of a Rankine vortex with free surface was also studied in a related work [13] using a simplified approach, which restricts the flow to a narrow gap along the bottom and lateral walls. This approach allowed to study in detail the interaction between gravity and KK waves, which was shown to generally give rise to an instability. This resonance was proposed to be at the origin of the sloshing phenomenon observed in some experiments [6]. The global stability results presented here suggest a second mechanism involving gravity and Rossby waves. The gravity-KK wave interaction is not revealed by global stability in the sample case presented in Figure 5 because the corresponding branches do not cross, but it certainly exists in other ranges of parameters. Additional efforts are needed to delimitate the range 
of existence of gravity/KK and gravity/Rossby interactions. Experimental results should also be reexamined to identify the footprint of the waves present in the setup and responsible for pattern formation.

\section{Summary and perspectives}

To conclude, this paper synthesized global stability results obtained for three models of rotating flows with a free surface, which are highly relevant for experiments with a rotating bottom. The first model, namely solid body rotation, is always stable but turns out to be academically interesting as inertial, gravity and Rossby waves can be found in this case. The second model consisting of a potential rotation admits two families of waves, namely gravity and centrifugal waves, whose interaction was proposed to be at the origin of the symmetry breaking appearing experimentally [1]. However, this mechanism is only suited for strong rotation rates. The third considered model, which is more relevant for smaller rotation rates, includes an inner core in solid body rotation and an outer potential region. It combines the physical mechanisms of the two first cases, and hence admits five kind of waves in the general case (which also includes the "dry" states) namely gravity, centrifugal, Rossby, KelvinKirchhoff and singular inertial. The interplay between all these families of waves, and their relevance to the experimentally observed patterns, will be the object of future investigations.

Acknowledgements. We thank the DGA for support and Tomas Bohr for numerous fruitful discussions.

\section{References}

[1] L. Tophøj, J. Mougel, T. Bohr, D. Fabre, The Rotating Polygon Instability of a Swirling Free Surface Flow, Phys. Rev. Lett. 110 (2013) 194502

[2] G.H. Vatistas, A note on liquid vortex sloshing and Kelvin's equilibria, J. Fluid Mech. (1990) 241-248

[3] R. Bergmann, L. Tophøj, T.A.M. Homan, P. Hersen, A. Andersen, T. Bohr, Polygon formation and surface flow on a rotating fluid surface, J. Fluid Mech. 679 (2011) 415-431

[4] K. Iga, S. Yokota, S. Watanabe, T.H. Niino Ikeda, N. Misawa, Submitted to Fluid Dyn. Res. (2014)

[5] T.R.N. Jansson, M.P. Haspang, K.H. Jensen, T. Bohr, Polygons on a Rotating Fluid Surface, Phys. Rev. Lett. 96 (2006) 174502

[6] T. Suzuki, M. Iima, Y. Hayase, Surface switching of rotating fluid in a cylinder, Phys. Fluids 18 (2006) 101701

[7] M. Rieutord, L. Valdettaro, Inertial waves in a rotating spherical shell, J. Fluid Mech. 341 (1997) 77-99

[8] J. Mougel, Étude de stabilité linéaire du seau de Newton, Mémoire de stage de Master, ISAE, 2011

[9] F. Hecht, New development in freefem++, J. Numer. Math. 20 (2012) 251-265

[10] H.P. Greenspan, The theory of rotating flows, Cambridge Univ. Press (1969)

[11] R.A. Ibrahim, Liquid Sloshing Dynamics, Theory and Applications, Cambridge Univ. Press (2005)

[12] R.A. Cairns, The role of negative energy waves in some instabilities of parallel flows, J. Fluid Mech. 92 (1979) $1-14$

[13] D. Fabre, J. Mougel, Generation of tree-dimensional patterns through wave interaction in a model of free surface swirling flow, Accepted for publication in Fluid Dyn. Res. (2014)

[14] P.G. Saffman, Vortex Dynamics, Cambridge Univ. Press, 1992 